\title{
Epidemiology of soccer players traumatic injuries during the 2015 America Cup
}

\author{
Osvaldo Pangrazio ${ }^{1}$ \\ Francisco Forriol $^{2}$ \\ 1 Comisión Médica Conmebol (Confederación Su- \\ damericana de Fútbol), Asunción, Paraguay \\ 2 Department of Clínica Sciences, University San \\ Pablo - CEU, Boadilla del Monte, Spain
}

Corresponding author:

Francisco Forriol

Department of Clínica Sciences, University San

Pablo - CEU

Campus Montepríncipe

28668 Boadilla del Monte, Spain

E-mail: fforriol@mac.com

\section{Summary}

Aim: to provide an overview of the traumatic injuries sustained by players in the 2015 America Cup.

Material and methods: we collected the medical reports on all the matches held during the 2015 America Cup, in Chile, in 2015. Twelve American teams took part in the championship, consisted of 26 matches with a total of $\mathbf{2 7 6}$ players. The physician for each team sent a request form of the traumatic injuries sustained, including the time at which the injury was produced, the location and diagnosis, its severity and the circumstances (contact injury, sanction, treatment required).

Results: the mean number of minutes played was 233 (SD: 147) (5-570) minutes. An injury occurred every 58 minutes, which means that there were 17.25 injuries per 1,000 minutes of match time. We found 44 injuries in 30 players. There were 14 non-contact injuries, and 30 contact injuries, of which 13 were declared fouls and resulted in cards being given. Five teams had one injured player, two had 2, two had 4, and one had 25 injuries. The most frequent injuries were those to the lower limbs. The muscles strains happened in the second part of the second half of the match, the ACL rupture at the end of the first half, and the other sprains and strains in the second half. The contusions occurred at all times throughout the match, although they seemed to be concentrated towards the end of the first half, while the cases of tendinitis were caused in the first part of the second half.

Conclusion: football injuries are very common, and even though serious injuries are rare, it is increasingly necessary to set protocols for action which ensure good medical attention at all levels to address the problems that arise, both during training and in competitions, and to be prepared to treat serious injuries if these occur.

KEY WORDS: soccer, athlete, sport, muscle, ligament, tendon.

\section{Introduction}

Football is the most popular sport in the world, with over 270 million participants ${ }^{1}$, and it is also the sport that is most frequently played by people under 18 years of $\mathrm{age}^{2}$. However, despite its importance and the high number of injuries sustained, many of which mean that people have to give up sport, there is little homogeneous data collection of sports injuries, and no internationally accepted prevention protocol.

Competitive sports events provide an opportunity for establishing protocols of action and for analyzing the cause and nature of the injuries that occur. Some lesions, particularly to the muscles and tendons, can be prevented by ensuring good muscle condition, but others that arise during particular circumstances of the match are inevitable, and it is then that action must be taken to bring about a full recovery as soon as possible. Although some programs have been developed to prevent such injuries ${ }^{3,4}$, which have been the object of widespread consensus and have been publicized and analyzed, they still lack evidence ${ }^{5,6}$. Finding out what happens in championships offers the best way of predicting and preventing injuries ${ }^{7}$. In the present study, we analyzed the injuries to players in the 2015 America Cup.

\section{Material and methods}

We collected the medical reports on all the matches held during the 2015 America Cup, in Chile, in June and July 2015. Twelve American teams took part in the championship, selected from a previous competition: Argentina, Bolivia, Brazil, Chile, Colombia, Ecuador, Paraguay, Peru, Uruguay and Venezuela, all South American members of COMEMBOL (South 
America Football Confederation) and two invited selections, Jamaica and Mexico, from Central America (CONCACAF). The championship had an initial phase with four teams in each of three groups, which played against each other in a league system, so that the first two went through to the final phase. The players' physical characteristics are displayed in Table 1. During the first phase, they played 18 matches, while the second phase consisted of 4 matches in the quarter-finals, 2 in the semi-finals, 1 match for third and fourth position, and the cup final, which meant that the entire championship consisted of 26 matches, all of which lasted 90 minutes, with the winner decided by penalties if necessary. Only the final was allowed extra time of $30 \mathrm{~min}$ utes. Each selection had 23 players, which made a total of 276 players in the whole championship. Of these, 35 were goalkeepers, 88 defenders, 89 midfielders and 61 forwards. Their mean age was 27 (17-37) years. The physician responsible for each team sent in a report on the traumatic injuries sustained. The form included the date and time of the match, as well as details about the doctor and his/her contact details in case further information was needed. Each report contained the player's number, the exact time (in minutes during the match) at which the injury was produced, the location and diagnosis of the injury, both indicated by code, and its severity in terms of the number of days' absence from training and match play $(0=$ no days of absence; grade $1=1$ day of absence; $2=2$ days of absence; 7 = one week; $14=2$ weeks, and 30, the highest degree of severity, with absence of more than 4 weeks). The injury was considered to be a contact lesion when there was contact with another play or object, except the ground.

The physicians also noted down the circumstances, indicating whether there was contact with other players and whether the referee imposed any sanction, and any consequences, that is, whether the referee gave a red or yellow card, and whether the player required medical treatment or physiotherapy, during or after the match. The codes indicated the position of the lesion in the following terms: trunk (1: head; 2 : neck; 3 : thoracic spine; 4: lumbar spine; 5 : sternum and ribs; 6 : abdomen, 7: pelvis and sacrum); upper limbs (11: shoul- der; 12: arm; 13: elbow; 14: forearm; 15: wrist; 16: hand; 17: fingers and 18: thumb); lower limbs (21: hip; 22: groin; 23: thigh; 24: knee; 25: shin; 26: Achilles tendon; 27: ankle; 28: foot and 29: toes). The codes for diagnosis were: 1 : concussion and loss of consciousness; 2: concussion without loss of consciousness; 3 : fracture; 4: dislocation; 5: muscular ruptures; 6: tendon strain; 7: ruptured ligament with instability; 8: ruptured ligament without instability; 9: meniscal injury; 10: sprain; 11: strain; 12: contusion; 13: bursitis; 14: tendinitis; 15: laceration or abrasion; 16: other).

We carried out a descriptive statistical analysis of each of the parameters analyzed, and compared the injuries in terms of the players' positions. Variables were expressed as means and standard deviation. The Kolmogorov - Smirnov test was used in order to assess distribution normality and the Student $t$ - test was used for the comparison of two groups. Differences $p<0.05$ were considered statistically significant. The study was conducted ethically according to international standards ${ }^{8}$.

\section{Results}

Goalkeepers were significantly taller $(p<0.05)$ than the other players, followed by defenders, forwards and lastly midfielders. Goalkeepers also weighed more than other players $(p<0.05)$. The BMI was similar in all players (Tab. 1).

We divided the players according to match time between those who played for under 90 minutes; between 91 and 180 minutes; between 181 and 270 minutes; between 271 and 360; between 361 and 450; and more than 451 minutes (Tab. 2). Fifty-eight players did not take part in any of the matches. The mean number of minutes played was 233 minutes (SD: 147), with a maximum of 570 minutes and a minimum of 5 minutes. By position, the goalkeepers were the players who played longest, since only 15 different goalkeepers participated in the entire championship (Tab. 3). The forwards played least (204 minutes, SD: 144), followed by the midfielders (220 minutes, SD: 146) and defenders (255 minutes, SD: 137) (Tab. 4).

Table 1. Global anthropometric characteristic of the soccer players and depending of the field position.

\begin{tabular}{llll}
\hline Field position & $\begin{array}{l}\text { Height X (SD) } \\
\text { Max - } \min \end{array}$ & $\begin{array}{l}\text { Weight X (SD) } \\
\text { Max - } \min \end{array}$ & $\begin{array}{l}\left.\text { BMI (weight/height }{ }^{2}\right) \\
\text { Max - } \min \text { X (SD) }\end{array}$ \\
\hline Global & $1.81(0.06)$ & $73.45(6.41)$ & $22.9(1.54)$ \\
& $1.96-1.60$ & $92-57$ & $27.42-18.92$ \\
\hline Goalkeepers & $1.85(0.06)$ & $80.19(6.58)$ & $23.42(1.77)$ \\
& $1.96-1.60$ & $92-63$ & $27.43-20.01$ \\
\hline Defenders & $1,80(0.06)$ & $73.71(5.35)$ & $22.79(1,44)$ \\
& $1.96-1.68$ & $87-60$ & $26.79-19.32$ \\
\hline Midfielders & $1.76(0.05)$ & $71.11(5.23)$ & $22.89(1.36)$ \\
& $1.88-1.64$ & $85-57$ & $26.7-19.52$ \\
\hline Forwards & $1.79(0.06)$ & $72.72(6.6)$ & $22.76(1.69)$ \\
& $1.90-1.64$ & $86-60$ & $26.95-18.92$ \\
\hline
\end{tabular}


Table 2. Players time played (minutes).

\begin{tabular}{llllll}
\hline Time played (minutes) & Goalkeepers & Defenders & Midfielders & Forwards & Global \\
\hline$<90$ & 23 & 32 & 28 & 21 & 104 \\
\hline $91-180$ & 2 & 11 & 19 & 17 & 49 \\
\hline $181-270$ & 3 & 21 & 20 & 11 & 53 \\
\hline $271-360$ & 5 & 15 & 3 & 3 & 39 \\
\hline $361-450$ & 0 & 2 & 8 & 5 & 8 \\
\hline$>450$ & 3 & 7 & & 23 \\
\hline
\end{tabular}

Table 3. Games played in relation with the field position.

\begin{tabular}{lllll}
\hline Games played & Goalkeepers & Defenders & Midfielders & Forwards \\
\hline 0 & 21 & 20 & 10 & 7 \\
\hline 1 & 2 & 19 & 8 & 6 \\
\hline 2 & 2 & 9 & 14 & 11 \\
\hline 4 & 3 & 23 & 23 & 12 \\
\hline 5 & 5 & 12 & 17 & 15 \\
\hline 6 & 0 & 5 & 3 & 4 \\
\hline
\end{tabular}

Table 4. Time played (minutes) in relation with the field position.

\begin{tabular}{ll}
\hline Field position & Minutes played \\
& X (SD) \\
& Max - min \\
& N \\
\hline Global & $233.66(147.12)$ \\
& $570-5$ \\
& 219 \\
\hline Goalkeepers & $322(149.18)$ \\
& $570-90$ \\
& 15 \\
\hline Defenders & $255.84(137.62)$ \\
& $570-15$ \\
& 68 \\
\hline Midfielders & $220.62(146.57)$ \\
& $569-10$ \\
\hline Forwards & 80 \\
& $204.59(144.06)$ \\
& $570-5$ \\
\hline
\end{tabular}

Injuries were reported in 13 out of the 28 matches. In 5 matches there was only one incident; in 2 matches there were 3 injuries; one match had 4 , two had 5 , two had 6, and one match resulted in 7 injuries (Fig. 1). An injury occurred every 58 minutes, which means that there were 17.25 injuries per 1,000 minutes of match time. There were 14 non-contact injuries, and 30 contact injuries, of which 13 were declared fouls and resulted in cards being given (Tab. 5). Two injuries were classified as fouls, but the referee did not give a card, while the other $50 \%$ was not indicated by the referees. The questionnaires reported 44 injuries in 30 players, 3 players had 2 injuries, one player had 3 and another had 9 injuries. From the point of view of position, 3 goalkeepers, 8 defenders, 10 midfielders and 9 forwards were injured. As to position, there were 7 head injuries, one in the ribs, one in the lumbar region, one in the shoulder, one in the forearm and two in the hand. The most frequent injuries were those to the lower limbs: 1 in the hip, 8 in the thigh, 5 in the knee, 6 in the shin, one affecting the Achilles tendon, 2 in the ankle and 8 injuries to the foot.

The injuries, which resulted in a player's withdrawal from the championship were a scaphoid fracture in the case of a goalkeeper who was kicked by a team-mate during a training session, a $\mathrm{m}$. rectus anterior rupture and a case of rupture of the anterior cruciate ligament with joint instability. The injuries were diagnosed as follows: one concussion without loss of consciousness, three sprains, two of the knee and one of the shoulder, nine muscle strains, two cases of tendinitis, one in the patellar ligament and the other affecting the Achilles tendon, and a case of metatarsalgia. Most of the players were able to take part in the next match, but some required more days of rest (Fig. 2). Of the players who were injured, 17 played for more than 270 minutes in the championship, 5 played for 
Table 5. Referees cards sanction during the championship.

\begin{tabular}{lllll}
\hline Field position & $\mathbf{1}$ yellow card & $\mathbf{2}$ yellow cards & $\mathbf{3}$ yellow cards & Red cards \\
\hline Goalkeepers & 3 & - & - & - \\
\hline Defenders & 29 & 6 & 1 & 4 \\
\hline Midfielders & 27 & 10 & 2 & 1 \\
\hline Forwards & 18 & 1 & - & 3 \\
\hline Total & 77 & 17 & 3 & 8 \\
\hline
\end{tabular}

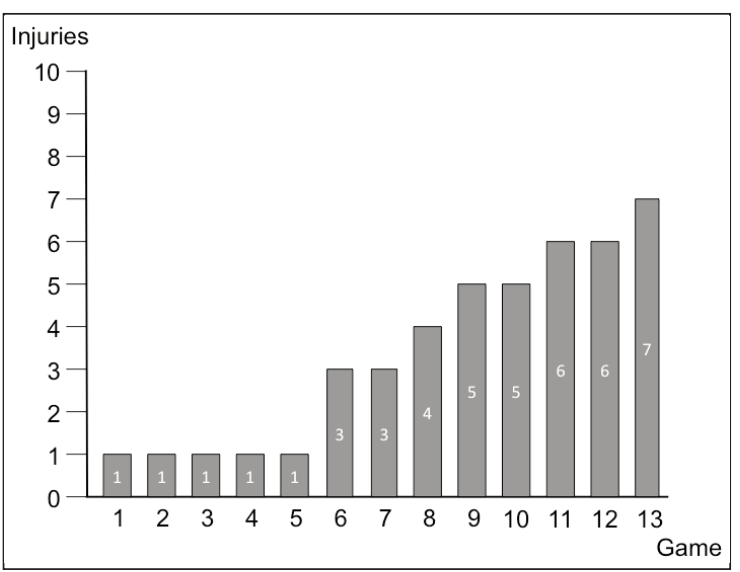

Figure 1. Injuries distribution per match

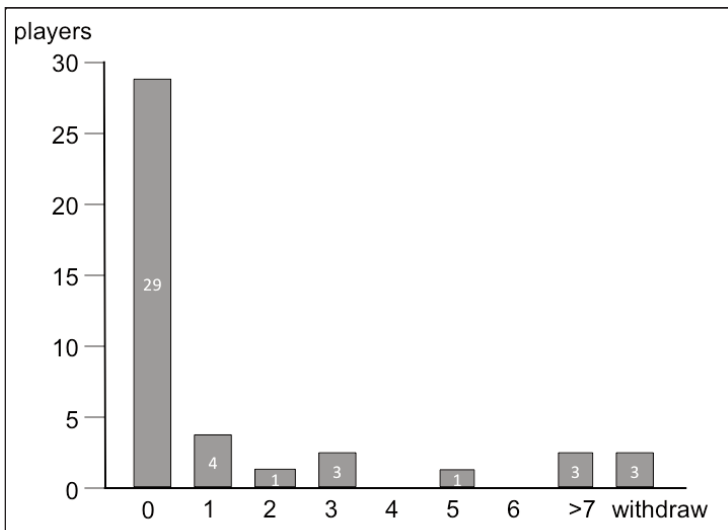

Figure 2. Injured players rest days

between 180 and 270 minutes and the rest (4) played under 180 minutes. The injured players saw 16 yellow cards and one red, and 7 of the injured players scored 14 of the goals in the championship.

Injuries occurred at many different points during the matches. The muscles strains happened in the second part of the second half of the match, the ACL rupture at the end of the first half, and the other sprains and strains in the second half. The contusions occurred at all times throughout the match, although they seemed to be concentrated towards the end of the first half, while the cases of tendinitis were caused in the first part of the second half. Muscular injuries were most frequent after one hour of play, but bruises occurred throughout the match, even though they tended to be more common towards the end of the first half (Fig. 3). From the perspective of the teams, there were major differences. Five teams had 1 injured player, two had 2, two had 4, and one had 25 injuries (Fig. 4).

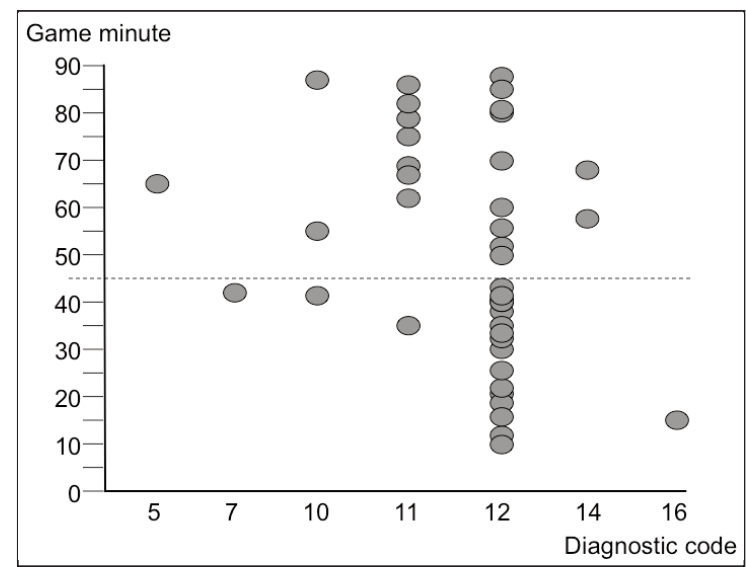

Figure 3. Injuries distribution during the match time (codes: 5 = muscle injuries; 7 : ligament rupture with instability; 10 : sprain; 11: strain; 12: contusion; 14: tendinitis; 16 : metatarsalgia)

\section{Discussion}

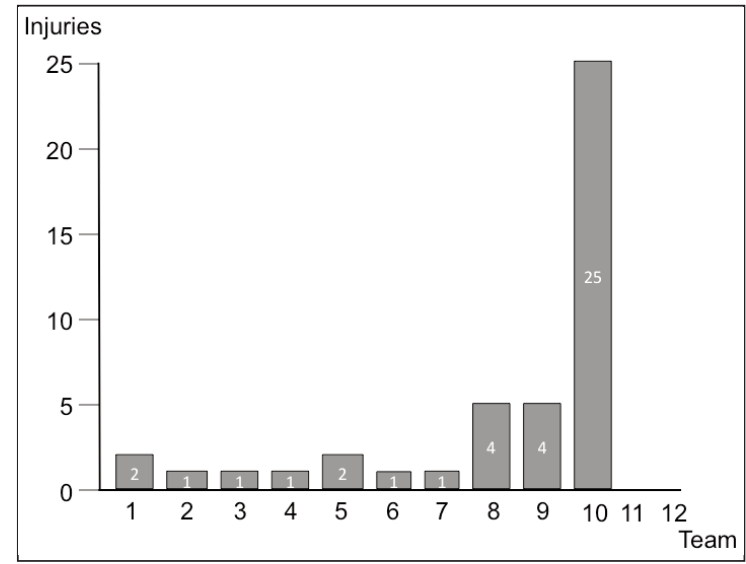

Figure 4. Total injuries per team 
Professional footballers are an heterogeneous group of sportsmen with very varied clinical antecedents ${ }^{9}$, and their injuries have a wide variety of causes. Players in a winning team have a greater risk of injury ${ }^{10}$. In all the teams taking part in the FIFA championships in 2002, 2006 and 2010, it was found that injuries were more frequent at certain points during the match: 5 minutes after the referee shows a red or yellow card, after another injury, or after a goal is scored. The most frequent time at which injuries occurred was during the last 15 minutes of the first half. In the 2015 America Cup we saw that the case of muscle strains happened in the second part of the second half of the match, the ruptured ACL at the end of the first half, the sprains and strains in the second half, bruises throughout the match, though commonly towards the end of the first half, while the two cases of tendinitis occurred in the first part of the second half.

The number of injuries is related to the number of fouls in a given match ${ }^{9}$; the incidence of injuries originating with fouls $(20.6 / 1,000$ hours of play) was lower than that of non-contact injuries, for all the positions on the pitch $^{11}$. In our study, injuries were reported in 13 of the 28 matches with great variation between matches; in three matches, 6 or 7 injuries were reported. There was also considerable variation between teams: two had no injuries in the entire championship, while one team alone had 25.

FIFA has systematically reported all the injuries occurring during its championships since 1998. Junge and Dvorak $^{12}$ analyzed 3,944 injuries, in 1,546 matches, between 1998 and 2012, equivalent to 2.6 injuries per match. Most injuries (80\%) were caused by contact, and $47 \%$ of these were due to a player breaking the rules. The 427 players in the 16 teams making up the first division of the Spanish league in 2008/2009, reported 1,293 injuries (145 of which were recurrent). The total incidence of injuries was 5.65 per 1,000 hours of play, and injuries were more frequent during matches than training $(43.53 / 3.55)^{13}$. In our study, there was an injury once every 58 minutes of play, or 17.25 injuries for every 1,000 minutes. Fourteen of the injuries occurred without contact, and 30 as a result of contact, of which 13 were identified as fouls and punished with cards. Two injuries were identified as fouls but no card was given, while the rest $(50 \%)$ did not draw the referee's attention.

There is unanimity concerning the location and diagnosis of the injuries. Muscle and tendon injuries are the most frequent kind. Injuries are very common, but the way in which they are assessed is very varied ${ }^{14}$. Training injuries are more common in the pre-season and tend to decline in the course of the season ${ }^{15}$ or during the first three months of the championship ${ }^{16}$. In 2015 America Cup, there were 7 head injuries, one to the ribs, one in the lumbar region, one in the shoulder, one in the forearm, and two in the hand. The most frequent injuries were to the lower limbs: one in the hip, 8 in the thigh, 5 in the knee, 6 in the shin, one affecting the Achilles tendon, two the ankle and 8 the foot.

The UEFA Medical Committee published 11-year fol- low-up (2001-2012) covering a total of 1,743 players, from 27 teams in 10 different countries. They found 8,029 cases of absence on the grounds of injury. Each player suffered a mean of two injuries per season, with 50 injuries per team. Injuries to the ligaments declined over the period of the study, while the incidence of muscle injuries and severe lesions remained unchanged. The overall incidence of lesions also remained stable ${ }^{17}$. During the championship, 17 of the injured players played for more than 270 minutes, 5 played between 180 and 270 minutes, and the rest (4 cases) played for fewer than 180 minutes. The injured players were shown 16 yellow cards and one red card, while 7 of the injured players scored 14 goals during the championship. Although we do not know whether injuries are related to the amount of time played, it is obvious that the more someone plays, the more possibilities that player has of being injured.

The position on the field gives rise to different injury patterns. Goalkeepers have special circumstances and tend to suffer acute injury to the adductor muscles and injuries resulting from overloading the hip and pubis; injuries in the hamstring muscles are rare ${ }^{18}$. Kristenson et al., ${ }^{19}$ took goalkeepers as their standard, and found that defenders had an index of 1.91, midfielders 1.78 and forwards 1.82. In our study it was the goalkeepers who played longest, since only 15 goalkeepers played throughout the entire championship. The forwards played least, followed by the midfielders, and the defenders. Moreover, 44 injuries were reported in 30 players, 3 players had 2 injuries, one had 3 , and another had 9 . According to position, 3 goalkeepers, 8 defenders, 10 midfielders and 9 forwards were injured.

Approximately half of the injuries meant that the player had to be absent from training and matches for one week, and a third required absence of 1 to 4 weeks. Around $10-15 \%$ of all lesions were assessed as serious. Between $2 \%$ and $20 \%$ of all football-related injuries are fractures, and one third of these are in the legs. In a retrospective study conducted in Belgium ${ }^{20}$, 1,600 fractures $(3 \%)$ were found in the lower limbs, the most frequent site being the ankle $(37 \%)$, followed by the foot $(33 \%)$ and the tibia (22\%). The most infrequent were fractures of the fibula $(9 \%)$. The incidence was higher in older players or amateurs than in younger players and professionals, although according to Faude et al., ${ }^{2}$ fractures are more common in players aged less than 15. Fractures of the upper limbs in young footballers in the Netherlands increased considerably between 1988 and 2009, from $19.4 \%$ in players aged 5 to 10 years, to $73.2 \%$ in players aged 11 to 14 , and $38.8 \%$ in those aged 15 to $18^{21}$. In the 2015 America Cup only one scaphoid fracture occurred, in a goalkeeper during training.

Muscular injuries are the most frequent kind among soccer players, but even though they are very common, the type of treatment prescribed and the recovery time allowed vary considerably. Hallen and Ekstrand 22 followed 89 European professional teams between 2001 and 2013. From 2007 they observed that a negative MR resulted in a shorter absence (6 days). The $\mathrm{m}$. biceps femoris was the location of $83 \%$ of the hamstring 
injuries, while $12 \%$ affected the $m$. semimembranous and $5 \%$ in the $m$. semitendinous. Recurrence was commoner in injuries to the femoral biceps (18\%). Groin pain resulted in a mean absence of 9 days, less than lesions of the hamstring muscles (13 days), quadriceps (12 days) and leg muscles (13 days). In the context of the UEFA register of incidents in European teams over eleven seasons, Ueblacker et al., ${ }^{23}$ followed 1,981 players from 31 football teams, and found 2,287 muscular injuries in the thighs, amounting to $25 \%$ of all lesions, $88 \%$ of which had been caused indirectly and $12 \%$ by contact; $60 \%$ of the indirect injuries and $76 \%$ of the direct ones had occurred during championship matches. The amount of leave required in the case of indirect injuries was also greater, namely 18.5 days as compared to 7 days in the case of direct injuries. Hägglund et al., ${ }^{24}$ analyzed muscular lesions in the UEFA register from 2001 to 2010, and identified 2,123 muscle injuries. Injuries to the adductors (56\%) and quadriceps $(63 \%)$ muscles were the most frequent injuries to the shooting leg. Intrinsic factors related to muscle injury were previous injury, age, and the leg used for kicking. Moreover, injuries varied across the season and depending on the player's position. Older players had twice as many lesions, and the dominant leg was also injured more often. Injuries to the quadriceps were more frequent in the pre-season, while the adductors, hamstrings and gastrocnemius muscles experienced more injuries during the season ${ }^{24}$. Prevention programs are also controversial. In a study of 20 teams with 296 players, the percentage of hamstring injuries was $6.2 \%$, and there were no differences with respect to the groups that had taken part in the NHE (Nordic Hamstring Exercise) prevention program ${ }^{25}$. During the championship we studied, muscle injuries were more frequent after one hour of play, while bruises occurred throughout, although they tended to be more usual in the first half, particularly towards the end.

Cerebral contusions are unusual in football, but when they do occur they may be serious. Concussion is frequent, but there is usually no loss of consciousness, and although the referees generally consider it to be a foul and take out a yellow card when players jump with their arms open, the most frequent injury resulting from this is a blow to the face. Nonetheless, the physician must act promptly in case there is loss of consciousness. It is therefore essential to diagnose how serious the concussion is and to avoid later consequences, so the player should only return to the match if this is safe. In cases of cerebral contusion the player should never play again on the same day, even if he has no symptoms and the clinical examination is normal ${ }^{26}$.

The knee joint suffers the most severe injuries in soccer, many of which require surgical treatment. Lundblad et al., ${ }^{27}$ described injury to the collateral medial ligament of the knee as the most frequent lesion among professional footballers. They found 346 injuries to the collateral medial ligament of the knee, $70 \%$ caused by contact, which had happened during 1,057,201 hours of play $(0.33 / 1,000 \mathrm{~h})$. The incidence of the injury during competition was 9 times more than during training.
The mean absence from training and match play was 23 days. Meniscal lesions were found to be located in the internal meniscus in $24 \%$ of cases, the external in $8 \%$, and associated with ligament injury in $20 \%$ to $30 \%$ of cases $^{28}$. Quisquater et al.,29 analyzed two seasons of Belgian football 10 years apart, and found 9,971 knee injuries, 5,495 in the earlier season (1999-2000) and 4,476 in the second one (2009-2010). They observed a significant drop in the number of injuries, which fell from $1.5 / 100$ players, in 2000 , to $1.2 / 100$ players in 2010. Of these knee injuries, $6 \%$ affected the $\mathrm{ACL}$, which rose slightly from the first season to the second, from 0.081 to 0.084 per 100 players. Little can be done to prevent ACL injuries. In the review carried out by Grimm et al., ${ }^{30}$ among 11,562 athletes, 7,889 were analyzed specifically for ACL injuries. No results were available concerning the efficacy of the prevention programs. The Swedish register of ACL injuries ${ }^{31}$ reveals a ratio of women/men of $42: 58$. The injury had occurred during a match in $36 \%$ of women and $49 \%$ of men. In patients with a minimum follow-up of 5 years, $3.3 \%$ had had further surgery. According to Zaffagnini et al., ${ }^{32}$ after $A C L$ reconstruction only $71 \%$ were still competing four years after surgery.

Half of the ankle injuries were sprains, mainly caused by fouls during the match, while ankle impingement is very unusual among professional footballers ${ }^{33}$. Achilles tendon injuries accounted for $3.8 \%$ of all lesions, and were more frequent in older players, with high recurrence when the rest period was short ${ }^{34}$.

International teams players have long, intense seasons, and so it is interesting to know more about the relationship between minutes played and injuries sustained. Bengston et al., ${ }^{35}$ collected prospective data on matches lost because of injury, in 27 teams for 11 seasons. The matches were grouped according to the number of days of rest before each match. The total number of muscle injuries was higher in league matches with $\leq 4$ days of recovery time, compared with $\geq 6$ days, especially in cases of hamstring and quadriceps muscles injuries. The more matches were played, the more the incidence of muscle injuries rose in the same sequence of matches, and this was accompanied by an increase in ligament injuries during training sessions. Football injuries are hard to foresee and prevent. They are very common, and even though serious injuries are rare, it is increasingly necessary to set protocols for action which ensure good medical attention at all levels to address the problems that arise, both during training and in competitions, and to be prepared to treat serious injuries if these occur. Although when we talk of football injuries, trauma always springs to mind first, we should not forget that medical conditions are as frequent or more so, and they also require proper attention.

\section{Conflict of interests}

The Authors declare that they have no conflict of interests regarding the publication of this paper. 


\section{References}

1. Herrero H, Salinero JJ, Del Coso J. Injuries among Spanish male amateur soccer players: a retrospective population study. Am J Sports Med. 2014;42:78-85.

2. Faude $O$, Rößler R, Junge A. Football injuries in children and adolescent players: are there clues for prevention? Sports Med. 2013;43:819-837.

3. Barengo NC, Meneses-Echávez JF, Ramírez-Vélez R, Cohen DD, Tovar G, Bautista JE. The impact of the FIFA 11+ training program on injury prevention in football players: a systematic review. Int J Environ Res Public Health. 2014;11:1198612000.

4. Hammes D, Aus der Fünten K, Kaiser S, Frisen E, Bizzini M, Meyer T. Injury prevention in male veteran football players - a randomised controlled trial using "FIFA 11+". J Sports Sci. 2015;33:873-881.

5. Mayo M, Seijas R, Alvarez P. Calentamiento neuromuscular estructurado como prevención de lesiones en futbolistas profesionales jóvenes. Rev Esp Cir Ortop Traumatol. 2014; 58: 336-342.

6. Bizzini M, Junge A, Dvorak J. Implementation of the FIFA 11+ football warm up program: how to approach and convince the Football associations to invest in prevention. Br J Sports Med. 2013;47:803-806.

7. Timpka T, Jacobsson J, Bickenbach J, Finch CF, Ekberg J, Nordenfelt L. What is a sports injury? Sports Med. 2014;44: 423-428.

8. Padulo J, Oliva F, Frizziero A, Maffulli N. Muscles, Ligaments and Tendons Journal. Basic principles and recommendations in clinical and field science research. MLTJ. 2013;3(4):250252.

9. Spahn G, Grosser V, Schiltenwolf M, Schröter F, Grifka J. Football as Risk Factor for a Non-Injury-Related Knee Osteoarthritis - Results from a Systematic Review and Metaanalysis. Sportverletz Sportschaden. 2015;29:27-39.

10. Ryynänen J, Junge A, Dvorak J, Peterson L, Karlsson J, Börjesson $M$. The effect of changes in the score on injury incidence during three FIFA World Cups. Br J Sports Med. 2013; 47:960-964.

11. Ryynänen J, Junge A, Dvorak J, et al. Foul play is associated with injury incidence: an epidemiological study of three FIFA World Cups (2002-2010). Br J Sports Med. 2013;47:986-991.

12. Junge A, Dvorak J. Injury surveillance in the World Football Tournaments 1998-2012. Br J Sports Med. 2013;47:782-788.

13. Noya Salces J, Gómez-Carmona PM, Gracia-Marco L, Moliner-Urdiales D, Sillero-Quintana M. Epidemiology of injuries in First Division Spanish football. J Sports Sci. 2014;32:12631270.

14. McCall A, Carling C, Nedelec M, et al. Risk factors, testing and preventative strategies for non-contact injuries in professional football: current perceptions and practices of 44 teams from various premier leagues. Br J Sports Med. 2014;48:13521357.

15. Noya Salces J, Gomez-Carmona PM, Moliner-Urdiales D, Gracia-Marco L, Sillero-Quintana M. An examination of injuries in Spanish professional soccer league. J Sports Med Phys Fitness. 2014;54:765-771.

16. Bollars $\mathrm{P}$, Claes $\mathrm{S}$, Vanlommel L, Van Crombrugge $\mathrm{K}$, Corten $\mathrm{K}$, Bellemans $\mathrm{J}$. The effectiveness of preventive programs in decreasing the risk of soccer injuries in Belgium: national trends over a decade. Am J Sports Med. 2014;42:577-582.

17. Ekstrand J, Hägglund M, Kristenson $\mathrm{K}$, Magnusson $\mathrm{H}$, Waldén $M$. Fewer ligament injuries but no preventive effect on muscle injuries and severe injuries: an 11-year follow-up of the UEFA Champions League injury study. Br J Sports Med. 2013;47: 732-737.

18. Eirale C, Farooq A, Bisciotti G, Chalabi H. How injuries affect rehabilitation workload in a National Team during an elite soccer event? An experience from the FIFA 2010 World Cup. J Sports Med Phys Fitness. 2013;53:192-197.

19. Kristenson K, Bjørneboe J, Waldén M, Andersen TE, Ekstrand J, Hägglund M. The Nordic Football Injury Audit: higher injury rates for professional football clubs with third-generation artificial turf at their home venue. Br J Sports Med. 2013;47:775-781.

20. Vanlommel L, Vanlommel J, Bollars $P$, et al. Incidence and risk factors of lower leg fractures in Belgian soccer players. Injury. 2013:44:1847-1850.

21. de Putter CE, van Beeck EF, Burdorf A, et al. Increase in upper extremity fractures in young male soccer players in the Netherlands, 1998-2009. Scand J Med Sci Sports. 2015; 25:462-466.

22. van Beijsterveldt AM, Stubbe JH, Schmikli SL, van de Port IG, Backx FJ. Differences in injury risk and characteristics between Dutch amateur and professional soccer players. J Sci Med Sport. 2015;18:145-149.

23. Melegati G, Tornese D, Gevi M, et al. Reducing muscle injuries and reinjuries in one Italian professional male soccer team. Muscles Ligaments Tendons J. 2014:3:324-330.

24. Ueblacker P, Mueller-Wohlfahrt HW, Ekstrand J. Epidemiological and clinical outcome comparison of indirect ('strain') versus direct ('contusion') anterior and posterior thigh muscle injuries in male elite football players: UEFA Elite League study of 2287 thigh injuries (2001-2013). Br J Sports Med. 2015;49: 1461-1465

25. Hägglund $M$, Waldén $M$, Ekstrand J. Risk factors for lower extremity muscle injury in professional soccer: the UEFA Injury Study. Am J Sports Med. 2013;41:327-335.

26. Feddermann-Demont N, Straumann D, Dvořák J. Return to play management after concussion in football: recommendations for team physicians. J Sports Sci. 2014;32:1217-1228.

27. Lundblad M, Waldén M, Magnusson H, Karlsson J, Ekstrand J. The UEFA injury study: 11-year data concerning $346 \mathrm{MCL}$ injuries and time to return to play. Br J Sports Med. 2013; 47:759-762.

28. Frizziero A, Ferrari R, Giannotti E, Ferroni C, Poli P, Masiero S. The meniscus tear. State of the art of rehabilitation protocols related to surgical procedures. Muscles Ligaments Tendons J. 2013;2:295-301.

29. Quisquater L, Bollars P, Vanlommel L, Claes S, Corten K, Bellemans $J$. The incidence of knee and anterior cruciate ligament injuries over one decade in the Belgian Soccer League. Acta Orthop Belg. 2013;79:541-546.

30. Grimm NL, Jacobs JC Jr, Kim J, Denney BS, Shea KG. Anterior cruciate ligament and knee injury prevention programs for soccer players: a systematic review and meta-analysis. Am J Sports Med. 2015;43:2049-2056.

31. Kvist J, Kartus J, Karlsson J, Forssblad M. Results from the Swedish national anterior cruciate ligament register. Arthroscopy. 2014;30:803-810.

32. Zaffagnini S, Grassi A, Marcheggiani Muccioli GM, et al. Return to sport after anterior cruciate ligament reconstruction in professional soccer players. Knee. 2014;21:731-735.

33. Waldén M, Hägglund M, Ekstrand J. Time-trends and circumstances surrounding ankle injuries in men's professional football: an 11-year follow-up of the UEFA Champions League injury study. Br J Sports Med. 2013;47:748-753.

34. Gajhede-Knudsen M, Ekstrand J, Magnusson H, Maffulli N. Recurrence of Achilles tendon injuries in elite male football players is more common after early return to play: an 11-year follow-up of the UEFA Champions League injury study. $\mathrm{Br} \mathrm{J}$ Sports Med. 2013;47:763-768.

35. van der Horst N, Smits DW, Petersen J, Goedhart EA, Backx FJ. The preventive effect of the nordic hamstring exercise on hamstring injuries in amateur soccer players: a randomized controlled trial. Am J Sports Med. 2015;43:1316-1323. 